\title{
Cooperative Control and Smart Procurement Of Naturally Generated Energy (SPONGE) for PHEVs
}

\author{
Florian Häusler*, Yingqi Gu ${ }^{\dagger}$, Wynita Griggs ${ }^{\dagger}$, Emanuele Crisostomi ${ }^{\ddagger}$, Ilja Radusch* and Robert Shorten ${ }^{\dagger \S}$ \\ *Fraunhofer Institute for Open Communication Systems, 10623 Berlin, Germany \\ ${ }^{\dagger}$ School of Electrical, Electronic and Communications Engineering, University College Dublin, Ireland \\ ${ }^{\ddagger}$ Department of Energy, Systems, Territory and Constructions Engineering, University of Pisa, Italy \\ Email: emanuele.crisostomi@unipi.it \\ ${ }^{\dagger}$ IBM Research, Dublin,Ireland
}

\begin{abstract}
Electric vehicles can potentially be the best means of transportation for improving air quality, provided that they are powered by electricity from natural gas or wind, water or solar power. In this paper we describe a simple cooperative algorithm that exploits the energy management units of Plug-in Hybrid Electric Vehicles (PHEVs) to absorb the expected forthcoming energy available from renewable sources. The proposed approach bridges the gap between mobility patterns and power grid constraints, and allows to prevent green energy from being wasted while at the same time reducing the complexity burden of the power grid to charge unexpected loads of electric vehicles. Simulation results are given to show the efficacy of the proposed method.
\end{abstract}

\section{INTRODUCTION}

The increasing electrification of the transportation fleet is opening previously unexplored possibilities for a synergistic collaboration between hitherto disconnected fields of the electric power grid, i.e., the smart grid, and the intelligent transportation network to achieve a system-level integrated optimisation.

Electric Vehicles (EVs) and Plug-in Hybrid Vehicles (PHEVs) have been seen by the power grid community as a potential threat to the power grid, since accommodating a not-fully predictable large load could ultimately cause thermal overload of some network components, low voltages at sensitive locations of the network and increase the chances of phase unbalances, see [1], [2] and [3]. At the same time, the potential of EVs and PHEVs to provide ancillary services to the grid (e.g., Vehicle-to-Grid (V2G) applications) were investigated in [4], [5], [6] and [7]. Finally, references [8], [9] and [10] investigate optimal charging of electric vehicle in the presence of intermittent power generation from renewable energy sources (e.g., solar and wind).

Note that most of the related literature, including the previous references, tend to consider separately the transportation viewpoint and the power grid one. This is due to the fact that the mobility needs of PHEV owners and the requirements of the power grid are obviously decoupled. On the other hand, in this work we take a completely different point of view, and formulate an optimisation problem that jointly takes into account both mobility patterns and also the concerns of the power grid, as better explained in the following section.

\section{A. Contribution}

The main contribution of this paper is to propose and evaluate a completely new paradigm to control the way in which hybrid vehicles discharge and recharge their batteries.

In particular, we propose that a central management service orchestrates the mode in which PHEVs travel, i.e., between the Internal Combustion Engine (ICE) and the Electric Mode (EM). In this way, it is possible to control the depletion of the batteries of a fleet of a PHEVs, and make it equal to any pre-specified quantity. Accordingly, the power grid can know, and decide, in advance the amount of the load required by the fleet of PHEVs. In particular, we control the depletion of the batteries of single vehicles in order to minimise an overall utility function that takes into account the cost of recharging the PHEVs, and in doing so encourages the use of energy generated from renewable sources for battery charging. The proposed framework allows us to achieve the following two main objectives:

- We use weather forecasts to predict the expected energy available from renewable sources, e.g., in the next 24 hours. Then, we make the PHEVs travel in EV mode for enough time to deplete their batteries in order to accommodate for the energy available from natural sources. In this way we prevent naturally generated energy from being wasted due to the absence of a consistent electrical load, or of alternative storage systems;

- In principle, it allows the smart grid to decide in advance (e.g., a day-ahead) the amount of energy that will be required to fully recharge the PHEVs, given that the battery discharge is controlled in a centralised fashion.

\section{Controlling The travel MOde OF A HYBRid VEHICLE}

Based on the mechanical architecture, PHEVs can be classified into three categories: parallel hybrids, series hybrids, and power-split hybrids. In the parallel configuration, both the engine and the electrical motor can individually or collaboratively drive the vehicle. In the series case, a single motor is 
used to drive the wheels, and it can be either supplied by a battery, or by a generator transforming the engine power into electrical power, or both. Finally, the power-split hybrids use a power-split mechanism (e.g., a planetary gear) to combine the two previous configurations. Examples of papers describing the modeling and the management strategies to drive PHEVs can be found in [11], [12], [13] and [14]. Despite several techniques have been developed to optimally manage the switching between the ICE and the electric mode, or the proportion of torque provided by each of the two units, usually the following criteria are used as hard constraints (see [12]):

1) The State of Charge (SOC) of the battery should never drop under a certain threshold (i.e., to avoid endangering the lifetime of the battery);

2) The driver input (accelerating and braking pedals) should be consistently executed, unless it conflicts with the first restriction;

3) The overall energy efficiency and emission levels should be optimised, as long as the first and the second constraints are not violated.

At the same time, another common practice is to decompose the load power (which generally varies in a random fashion during real operations due to accelerations, decelerations, and climbing up and down grades) into a steady (average) power, and into a dynamic power with a zero average (see for instance [11]). Then, the common strategy is to use the ICE to supply the average power (with the advantage that it is possible to optimally configure the ICE to work very close to its most efficient working point), and the electric motor to supply the dynamic power. In this way, the total energy output from the dynamic powertrain is zero (on average) at the end of each driving cycle.

Some commercial vehicles do not allow the driver to bypass the existing Energy Management Unit (EMU) to override the optimally configured mechanism to switch between the two modes. However, manual switches are convenient for a number of reasons, e.g., to drive in some sensitive spots of a city in electric mode to avoid excessive pollution, as in Low emission zones (LEZ). Finally, refer to [15] as a practical example where the driving mode of a Toyota Prius had been remotely operated to control (via a Smartphone application) where to emit pollution due to ICE driving mode. In this paper, we use the same approach of ([15]) to switch the driving mode of a hybrid vehicle, but the goal is now to monitor the depletion of the battery to match the expected energy generated from renewable sources.

\section{A. Analogies with Demand Side Management techniques}

Our main idea here is to encourage the use of PHEVs in electric mode when it is expected that energy from renewable sources will be available soon. Note that similar ideas have been already applied to other smart electric domestic appliances (e.g., washing machines, tumble driers, dishwashers) in the context of so called "Demand Side Management" (DSM) techniques. In this case, controllable loads (i.e., loads that do not need to be operated with hard time constraints) are postponed to match favourable time slots, e.g., when PhotoVoltaic (PV) roof panels provide electrical supply, see for instance [16].

In this way, locally low-cost generated energy is prioritised over more expensive, possibly less environmentally friendly energy bought from the outer electrical grid. From this perspective, our approach extends typical DSM practices to the transportation field.

\section{THE SPONGE PARADIGM}

\section{A. Smart Procurement of Energy: SPONGE}

Let us consider a scenario in which a group of PHEVs, with maximum number of $N$, will participate in a SPONGE charging programme. For conveniences, we discretise every $k$ 'th day (a 24 hour day) into $T$ same time clock periods, each of length $\Delta T$. We assume that for some fixed period (e.g. $11 \mathrm{pm}$ to $6 \mathrm{am}$ ) during the day, these vehicles are plugged in, and that for this period, a reliable day-ahead forecast of available renewable energy is available. We denote this available energy by $E_{a v}(k+1)$. We assume that during some other fixed time period of the day (e.g. 9am to 6pm), these vehicles will proactively adjust their energy consumption patterns at every available clock period so as to make available space in the electric vehicle battery. We assume that this fixed time period consists of $M$ clock periods and we index every available clock period as $\tau \in\{1,2, \ldots, M\}$. Let us now denote the electric energy dissipated by the $i^{\prime}$ th vehicle at the $\tau^{\prime}$ th time clock period by $D_{i}(\tau)$. Our objective is to ensure that

$$
\sum_{\tau=1}^{M} \sum_{i=1}^{n(\tau)} D_{i}(\tau) \geq E_{a v}(k+1),
$$

As stated, the problem is a regulation problem that is depicted in Figure 1. Under ideal circumstances, a central authority computes the desired electrical energy consumption, and then broadcasts some signal which is received by vehicles to orchestrate the switching between EV and ICE mode, so as to satisfy the regulation constraint. For instance, the signal can be the probability to travel in EV mode rather than in ICE mode, or can be the proportion of the traction torque that should be provided by the EV engine rather than from the ICE engine. We shall denote the problem expressed by Equation 1 as the basic SPONGE problem.

\section{B. Smart Procurement of Energy: Exact SPONGE}

In some cases, the objective can be to make PHEVs travel in EV mode until they deplete their batteries in order to exactly match the expected energy that will be available from renewable sources. We shall denote this problem as "exact SPONGE", and its mathematical formulation is as follows:

$$
\sum_{\tau=1}^{M} \sum_{i=1}^{n(\tau)} D_{i}(\tau)=E_{a v}(k+1) .
$$




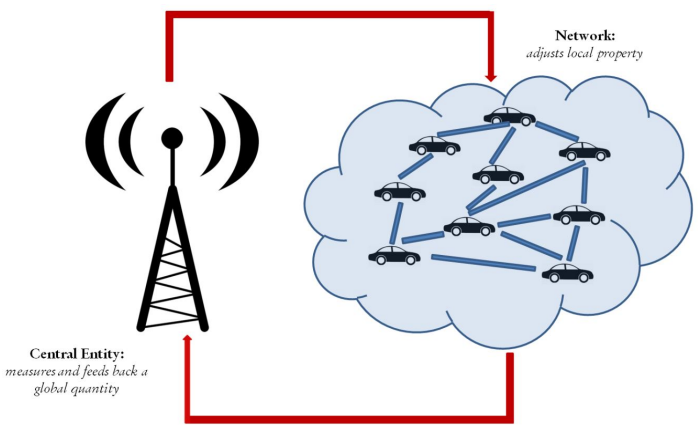

Fig. 1. Feedback loop for energy dissipation problem

The main advantage of the exact SPONGE approach is that when the fleet of vehicle connect to the grid for recharging, the quantity of required energy is already known in advance (i.e., it is equal to the expected energy available from renewable sources).

\section{Optimised access: Optimal SPONGE}

In some situations, certain vehicles may have prioritised access to the oncoming energy $E_{a v}(k+1)$ via some utility function $f_{i}$. Thus, the above problem can be reformulated in an optimisation framework as:

$$
\begin{cases}\begin{array}{cl}
\text { maximise } \\
\text { (or minimise) }
\end{array} & \sum_{i=1}^{n(\tau)} f_{i}\left(\bar{D}_{i}(\tau)\right) \\
\text { subject to } & \sum_{i=1}^{n(\tau)} \bar{D}_{i}(\tau)=\frac{E_{a v}(k+1)}{M} .\end{cases}
$$

where $\bar{D}_{i}(\tau)$ is defined as the average of energy distributed to the $i$ 'th vehicle up to time clock $\tau$. In addition, we assume that the available energy provided at every time clock $\tau$ equals $\frac{E_{a v}(k+1)}{M}$, which integrals during these $M$ clock periods gives the total available energy $E_{a v}(k+1)$. This optimisation may be solved in many ways under suitable assumptions on the $f_{i}$ 's. The problem is most interesting when the the $f_{i}^{\prime} s$ represent a generalised notion of utility (in which case the interest in Equation (3) is in maximisation) or a price that the $i$ 'th car pays (in which case one is interested in minimising the sum of utility functions) and is considered to be private information, not to be revealed to the utility or to other vehicles. The problem is then to solve the utility optimisation problem in a privacy preserving manner. Note that the $f_{i}$ 's may be incorporated to represent various use cases. Some interesting examples include the following.

(i) For example, Original Equipment Manufacturers (OEM's) may partner with utilities to provide a service where the price of energy is part of PHEV's owners car purchase plans. Those paying more upfront, may have prioritised access to 'free energy' as it becomes available. (ii) The $f_{i}$ 's could represent the price paid by an individual vehicle owner for energy access.

(iii) Or, they could be used to penalise vehicles with a lower load factor (fewer passengers).

(iv) They could be used to penalise vehicles that drive close to schools, hospitals, etc.

(v) Another interesting scenario is as follows. Some hybrid modes blend the EV motor with the ICE to optimise fuel economy/emissions. An interesting embodiment of the optimisation scenario is to take the required energy in a manner that minimises the impact on fuel economy of the fleet.

With regard to the SPONGE formulation several comments are appropriate.

Comment 1: Note that the SPONGE solution has the potential to simplify the "charging paradigm". Hitherto, most charging research has focussed on how to share the available energy among vehicles in a manner that is compliant with the desires of the EV owners, the constraints of the grid, and the available power. In this case, there might arise some problems in the power grid to accept the unexpected load, with the ultimate possibility of causing thermal overload, low voltages at sensitive locations of the network, and increased phase unbalance ([3]). Even ignoring this, the required optimisations often place severe constraints on the EV owners in the form of inconvenient charging profiles. On the other hand, in the solution of Problem (2), one would compute the same quantity in advance, and deplete the batteries of the vehicles while travelling of the same quantity. Thus, the charging process becomes fully schedulable and programmable. The charging problem can be reduced to a best-effort problem where the cars share the available energy during the charging period using some simple algorithm such as Additive Increase Multiplicative Decrease (AIMD) algorithms ([6], [17]). Thus, clearly, the difficulties of matching the demand and the offer are shifted to the driving stage through an optimal orchestration of the ICE and EV engines.

Comment 2: The discerning reader may ask why the individual vehicle owners should not simply expend the electric energy completely before switching to ICE mode. There are many reasons for doing this. First, in some engines, electrical power and ICE are combined to reduce overall consumption, or for other objectives of interest (e.g., extend the lifetime of the battery, as in [18]). Second, access to LEZs may be restricted to zero emission vehicles. Thus, maintaining a store of electrical energy for this purpose is also advantageous. Finally, depleting the battery beyond the energy levels available during the next charging period, may lead to a situation where the battery is not filled during the $k+1$ 'th charging period. Thereby, the ICE may need to be 
engaged prematurely in driving, thus leading to unnecessary emissions and increased fuel consumption.

Comment 3: Note that in some cases, depending on the number of vehicles on the road, the previous optimisation problems might not have a feasible solution. For instance, in the particular case that there are no vehicles on the road, PHEVs can not deplete their batteries to make room for the forthcoming energy. In such cases, we will be interested in a 'best-effort' solution, where the closest feasible solution is achieved instead, see for instance ([6]).

\section{AIMD ALGORITHM}

The third scenario illustrated in Section III-C is different from the previous two, since different probabilities should be computed for different users, taking personal constraints into account. Section III-C lists a number of candidate utility functions to represent the convenience (or the inconvenience) of the owners in travelling in a given mode. For the sake of simplicity, we assume from now on that the utility functions are convex functions that represent the inconvenience of owners in travelling in EV mode, and that they can be represented by equations $f_{i}\left(\bar{D}_{i}(\tau)\right)$, where $\bar{D}_{i}(\tau)$ represents the average energy consumed by the $i$ 'th vehicle up to time step $\tau$. Also, other utility functions can be used as well, as already remarked in Section III-C. Finally, a similar discussion can be made in terms of discomfort of travelling in ICE mode.

Such an optimal SPONGE scenario allows the central infrastructure to explicitly take into account personal needs of PHEVs' owners and there are many ways to solve the mathematical problem that arises. In this paper, we formulate the optimisation problem as a regulation problem with constraints, and we adopt an AIMD-like algorithm to solve it ([15], [19]). The main advantage of such an approach is that it can be implemented in a truly distributed manner (i.e., without requiring information exchange among the PHEVs), with moderate communication requirements.

The AIMD algorithm can be formulated as follows:

$$
\begin{array}{ll}
\text { if } & \sum_{h=1}^{\tau} \sum_{i=1}^{n(h)} D_{i}(h)<\frac{E_{a v}(k+1)}{M} \cdot \tau, \forall \tau=1, \ldots, M \\
\text { then } & p_{i}^{E V}(\tau+1)=\min \left\{p_{i}^{E V}(\tau)+\alpha, 1\right\}, \forall i=1, \ldots, n(\tau) \\
\text { elseif } & \sum_{h=1}^{\tau} \sum_{i=1}^{n(h)} D_{i}(h) \geq \frac{E_{a v}(k+1)}{M} \cdot \tau, \forall \tau=1, \ldots, M \\
\text { then } & \text { with probability prob } b_{i}^{E V} \\
& p_{i}^{E V}(\tau+1)=\beta p_{i}^{E V}(\tau), \forall i=1, \ldots, n(\tau) \\
& \text { or with probability } 1-\operatorname{prob}_{i}^{E V} \\
& p_{i}^{E V}(\tau+1)=\min \left\{p_{i}^{E V}(\tau)+\alpha, 1\right\}, \forall i=1, \ldots, n(\tau)
\end{array}
$$

The rationale of the algorithm is the following: some central entities are targeting to distributed the current available energy $\frac{E_{a v}(k+1)}{M}$ to the virtual battery of the set of vehicles at each time step $\tau$, in order to match the expected available energy from renewable sources at the end of the travelling stage (e.g., at the end of the day). If the overall dissipated energy of all PHEVs up to time clock $\tau$ is smaller than the desired one (i.e., $\sum_{h=1}^{\tau} \sum_{i=1}^{n(h)} D_{i}(h)<\frac{E_{a v}(k+1)}{M} \cdot \tau$ ), then each PHEV increases its probability $p_{i}^{E V}$ of travelling in EV mode (or alternatively, the proportion of torque provided by the electric engine) additively by a quantity $\alpha$ at next time clock $\tau+1$. Otherwise, if the overall dissipated energy of all PHEVs up to time clock $\tau$ is bigger than the desired one (such an event is often denoted as congestion event), the vehicles decrease their probability to travel in EV mode by a multiplicative factor $\beta<1$ with probability $\operatorname{prob}_{i}^{E V}$, or keep increasing the probability of travelling in EV mode with probability $1-\operatorname{prob}_{i}^{E V}$. Since at every time step $\tau$ either an Additive Increase or a Multiplicative Decrease step is performed, these algorithms are denoted as AIMD [20]. It can be proved that if all vehicles have the same parameters $\alpha, \beta$ and $\operatorname{prob}_{i}^{E V}$, then the SPONGE problem is solved by assigning the same probability (on average) to travel in EV mode to all vehicles. On the other hand, as proved in [19], by giving a different probability

$$
\operatorname{prob}_{i}^{E V}=\gamma \frac{\partial f\left(\bar{D}_{i}(\tau)\right) / \partial \bar{D}_{i}(\tau)}{\bar{D}_{i}(\tau)}, \forall i=1, \ldots, n(\tau),
$$

then the solution of the optimal SPONGE problem is achieved, provided that the utility functions $f_{i}(\cdot)$ have particular properties (e.g., they are concave is one is interested in maximising their sum, or they are convex if one is interested in minimising their sum, as in the case of interest here). Equation (4) simply states that the probability to back-off at a congestion event should be proportional to $f_{i}^{\prime}\left(\bar{D}_{i}(\tau)\right) / \bar{D}_{i}(\tau)$, and $\gamma$ is the proportionality factor required to map the ratio into a probability. Reference ([19]) also shows that achieving the optimal solution corresponds to achieving a consensus on the values of the derivatives of the single utility functions. Note that in order to apply the proposed AIMD method, the vehicles only need to know their own utility functions $f_{i}(\cdot)$, and communication requirements are limited to a broadcast from the central agent when $\sum_{h=1}^{\tau} \sum_{i=1}^{n(h)} D_{i}(h) \geq \frac{E_{a v}(k+1)}{M} \cdot \tau$ and a back-off step is required (i.e., no need of Vehicle-to-Vehicle communication). An application of the proposed algorithm is illustrated in detail in the next Section.

\section{Simulations}

Brief simulations are performed using the popular mobility simulator SUMO ([21]) and the given TRACI interface a a realistic road network imported from OpenStreetMap. Figure 2 shows the results for the first two algorithms. They refer to a scenario with about 600 PHEVs and a time period of 1000 seconds with 4 time windows of 250 seconds each. Vehicles that are running out of fuel, or whose battery is getting close to physical constraints are automatically discarded. The simulation refers to a very simple example, and might correspond to the case when employees go to work using their PHEVs, and the infrastructure (which could be the employer who provides charging stations, a smart grid operator, etc.) regulates the driving mode in order to meet the target of energy that will be available at the workplace. 

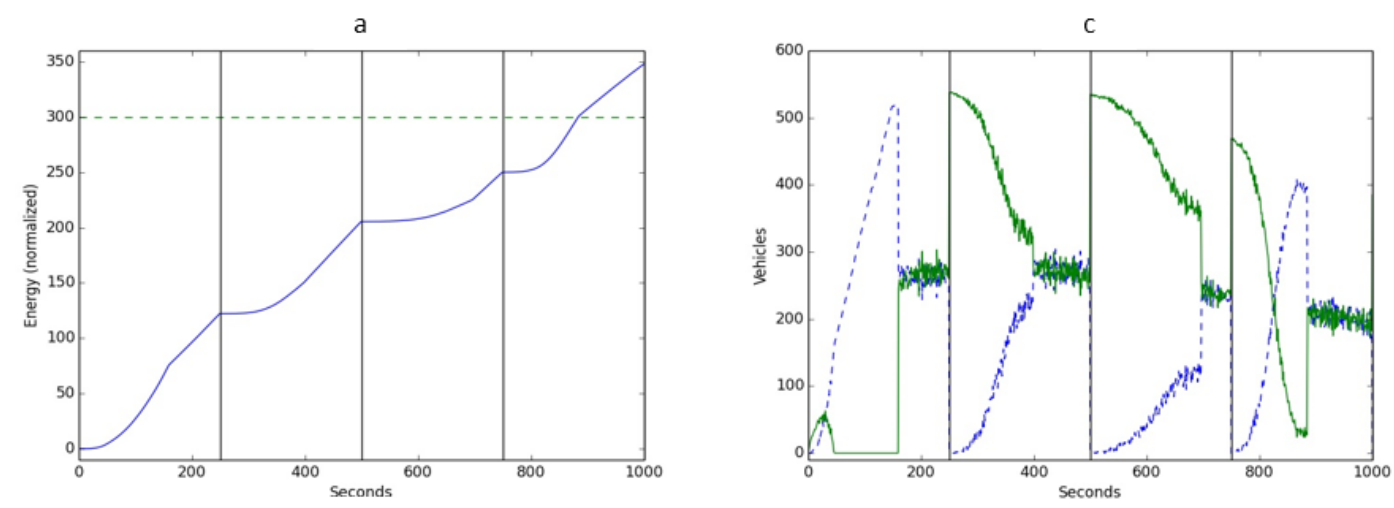

b
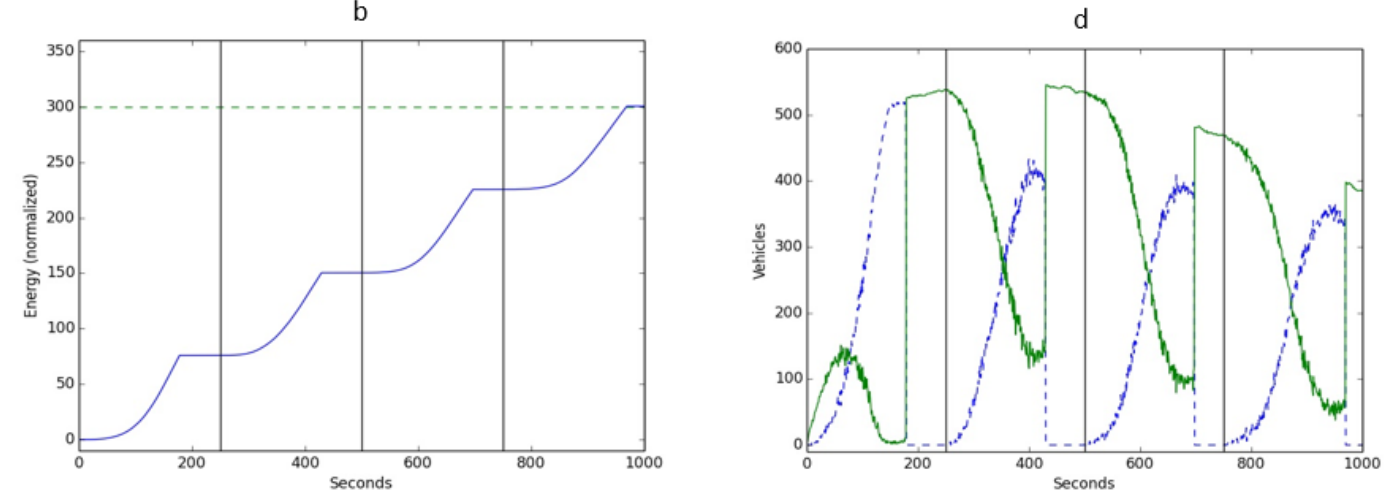

Fig. 2. Figures on the left show the total space for energy in the fleet of PHEVs in the two cases, in solid line, while the available energy from the grid is shown with the dashed line. Figures on the right show the mode in which the vehicles drive in the two cases to achieve the desired goal (dashed blue is EV mode, solid green is ICE mode). The vertical lines separate the time windows.

Simulation results referring to the third scenario (utility optimisation) are shown in Figures 3. We assumed that the inconvenience of vehicles in travelling in EV mode could be described through a convex quadratic function $f_{i}\left(\bar{D}_{i}\right)=$ $a_{i} \bar{D}_{i}^{2}+b_{i} \bar{D}_{i}$. Parameters $a_{i}$ and $b_{i}$ were different for every vehicle and randomly chosen in the interval $[0,1]$.

\section{CONCLUSION}

In this paper we have presented a new idea that takes advantage of the ability of PHEVs to both travel in electric and in fuel mode to absorb naturally generated electrical energy in a smart manner from the grid. From a theoretical perspective, such a problem can be easily formulated and solved using well-known algorithms for sharing a task among a number of distributed agents, (e.g., AIMD algorithms as in [17], [19]). From a practical point of view, the technology to remotely control the driving mode is also already available, as it was developed in ([15]) for different purposes.

Our current plan is to extend the preliminary simulation results given in Section $\mathrm{V}$ to more realistic and large-scale examples. In parallel, we intend to start implementing the approach in a reduced number of PHEVs, as a proof-ofconcept of the paper idea. We shall adapt the experimental set-up of ([15]) to the new case of interest, to remotely control the engine switching. The practical implementation of the algorithm will require a careful handling of possibly frequent mode switches, and averaging techniques will be used to implement them in a manner that would not endanger the life of the battery. Finally, we shall integrate a reliable weather forecast software, in order to take optimal decisions about when to switch from one mode to another mode.

\section{ACKNOWLEDGMENT}

This work was supported in part by Science Foundation Ireland grant 11/PI/1177.

\section{REFERENCES}

[1] O. Sundström and C. Binding, "Flexible charging optimization for electric vehicles considering distribution grid constraints," IEEE Transactions on Smart Grid, vol. 3, no. 1, pp. 26-37, 2012.

[2] Y. Cao, S. Tang, C. Li, P. Zhang, Y. Tan, Z. Zhang, and J. Li, "An optimized EV charging model considering TOU price and SOC curve," IEEE Transactions on Smart Grid, vol. 3, no. 1, pp. 388-393, 2012.

[3] J. de Hoog, T. Alpcan, M. Brazil, D. A. Thomas, and I. Mareels, "Optimal charging of electric vehicles taking distribution network constraints into account," IEEE Transactions on Power Systems, vol. 30, no. 1, pp. 365-375, 2015.

[4] E. Sortomme and M. A. El-Sharkawi, "Optimal scheduling of vehicle-togrid energy and ancillary services," IEEE Transactions on Smart Grid, vol. 3, no. 1, pp. 351-359, 2012.

[5] Y. He, B. Venkatesh, and L. Guan, "Optimal scheduling for charging and discharging of electric vehicles," IEEE Transactions on Smart Grid, vol. 3, no. 3, pp. 1095-1105, 2012.

[6] S. Stüdli, E. Crisostomi, R. Middleton, and R. Shorten, "Optimal real-time distributed V2G and G2V management of electric vehicles," International Journal of Control, vol. 87, no. 6, pp. 1153-1162, 2014. 

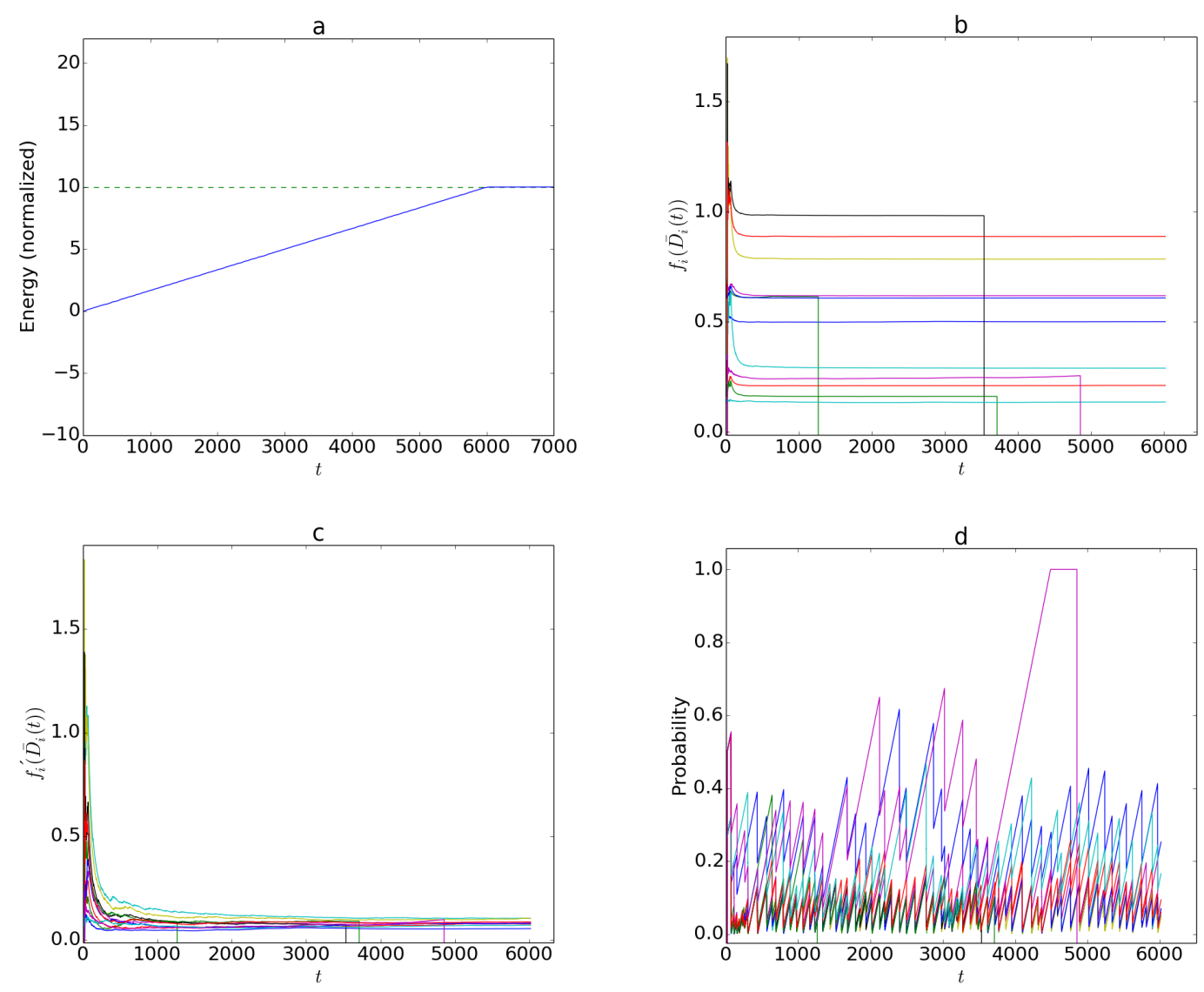

Fig. 3. Figure a shows that the overall constraint is again exactly satisfied at the end of the simulation. However, it is now achieved by minimising the sum of utility functions (single utility functions are shown in b). Figure c shows that the optimal solution has been achieved in fact, since there is a consensus on the derivative of the utility functions. Figure d emphasises that the utility optimisation problem is solved by assigning different probabilities of travelling in EV mode to different vehicles, according to their utility functions. Note that in Figure 3.d the probabilities increase additively, then drop at some congestion events, giving rise to saw-tooth signals that are characteristic of the AIMD algorithms. Furthermore, note that some vehicles reaching their destination before the end of the simulation exit the SPONGE programme in advance.

[7] S. Stüdli, W. Griggs, E. Crisostomi, and R. Shorten, "On optimality criteria for reverse charging of electric vehicles," IEEE Transactions on Intelligent Transportation Systems, vol. 15, no. 1, pp. 451-456, 2014.

[8] S. Gao, K. T. Chau, C. Liu, D. Wu, and C. C. Chan, "Integrated energy management of plug-in electric vehicles in power grid with renewables," IEEE Transactions on Smart Grid, vol. 63, no. 7, pp. 3019-3027, 2014.

[9] T. Zhang, W. Chen, Z. Han, and Z. Cao, "Charging scheduling of electric vehicles with local renewable energy under uncertain electric vehicle arrival and grid power price," IEEE Transactions on Smart Grid, vol. 63, no. 6, pp. 2600-2612, 2014.

[10] C.-T. Li, C. Ahn, H. Peng, and J. Sun, "Synergistic control of plug-in vehicle charging and wind power scheduling," IEEE Transactions on Smart Grid, vol. 28, no. 2, pp. 1113-1121, 2013.

[11] M. Ehsani, Y. Gao, and J. M. Miller, "Hybrid electric vehicles: Architecture and motor drives," Proceedings of the IEEE, vol. 95, no. 4, pp. 719-728, 2007.

[12] Y. Zhang, H. Liu, and Q. Guo, "Varying-domain optimal management strategy for parallel hybrid electric vehicles," IEEE Transactions on Vehicular Technology, vol. 63, no. 2, pp. 603-616, 2014.

[13] F. Yan, J. Wang, and K. Huang, "Hybrid electric vehicle model predictive control torque-split strategy incorporating engine transient characteristics," IEEE Transactions on Vehicular Technology, vol. 61, no. 6, pp. 2458-2467, 2012.

[14] J. Liu and H. Peng, "Modeling and control of a power-split hybrid vehicle," IEEE Transactions on Control Systems Technology, vol. 16, no. 6, pp. 1242-1251, 2008.

[15] A. Schlote, F. Häusler, T. Hecker, A. Bergmann, E. Crisostomi,
I. Radusch, and R. Shorten, "Cooperative regulation and trading of emissions using plug-in hybrid vehicles," IEEE Transactions on Intelligent Transportation Systems, vol. 14, no. 4, pp. 1572-1585, 2013.

[16] Y. Zong, L. Mihet-Popa, D. Kullmann, A. Thavlov, O. Gehrke, and H. W. Bindnern, "Model predictive controller for active demand side management with PV self-consumption in an intelligent building," in $3^{\text {rd }}$ IEEE PES Innovative Smart Grid Technologies Europe (ISGT Europe), Berlin, Germany, 2012.

[17] E. Crisostomi, M. Liu, M. Raugi, and R. Shorten, "Plug-and-play distributed algorithms for optimised power generation in a microgrid," IEEE Transactions on Smart Grid, vol. 5, no. 4, pp. 2145-2154, 2014.

[18] M. L. Shaltout, D. Chen, A. A. Malikopoulos, and S. Pannala, "Multi-disciplinary decision making and optimization for hybrid electric propulsion systems," in IEEE International Electric Vehicle Conference (IEVC), Florence, Italy, 2014.

[19] F. Wirth, S. Stüdli, J. Y. Yu, M. Corless, and R. Shorten, "Nonhomogeneous place-dependent Markov Chains, unsynchronised AIMD, and network utility maximization," 2015. [Online]. Available: http://arxiv.org/pdf/1404.5064v2.pdf

[20] D. Chiu and R. Jain, "Analysis of the increase/decrease algorithm for congestion avoidance in computer networks," Journal of Computer Networks, vol. 17, pp. 1-14, 1989.

[21] D. Krajzewicz, M. Bonert, and P. Wagner, "The open source traffic simulation package SUMO," in RoboCup 2006 Infrastructure Simulation Competition, Bremen, Germany, 2006. 